\title{
Fougères et autres éléments végétaux associés aux casse-têtes kanak ou l'art de communiquer sans parole
}

\section{Christian Coiffier}

\author{
(2) OpenEdition \\ Journals \\ Édition électronique \\ URL : http://journals.openedition.org/jso/6989 \\ DOI : $10.4000 /$ jso. 6989 \\ ISSN : $1760-7256$ \\ Éditeur \\ Société des océanistes \\ Édition imprimée \\ Date de publication : 15 octobre 2013 \\ Pagination : 133-147 \\ ISBN : 978-2-85430-035-2 \\ ISSN : 0300-953x
}

Référence électronique

Christian Coiffier, « Fougères et autres éléments végétaux associés aux casse-têtes kanak ou l'art de communiquer sans parole », Journal de la Société des Océanistes [En ligne], 136-137 | 2013, mis en ligne le 14 novembre 2013, consulté le 15 avril 2020. URL : http://journals.openedition.org/jso/6989 ; DOI : https://doi.org/10.4000/jso.6989 


\title{
Fougères et autres éléments végétaux associés aux casse-têtes kanak ou l'art de communiquer sans parole
}

par

\author{
Christian COIFFIER $^{*}$
}

\section{RÉSUMÉ}

Quelques casse-têtes kanak conservés dans divers musées présentent des bouquets de végétaux ligaturés sur leur manche. L'étude menée dans divers musées européens montre que les frondes de variétés particulières de fougères sont les plus fréquentes dans ces assemblages. La question se pose de connaître la raison de l'utilisation de ces fougères plutôt que d'autres.

Mots-clés : fougère, casse-tête, Kanak, NouvelleCalédonie, message non verbal, feu, incendiaire

"L'usage de ces bouquets dépasse d'ailleurs le sujet de la guerre, car on en compose pour symboliser tous les grands événements de la vie; et leur connaissance est nécessaire pour lire au livre où le peuple canaque inscrit ses messages. » (Leenhardt, $1930: 46$ )

Au XIX ${ }^{\mathrm{e}}$ siècle, les casse-têtes kanak présentaient fréquemment sur leur manche des enveloppements végétaux divers avec, parfois, l'adjonction de bouquets de plantes ligaturés sur ceux-ci. L'entretien insuffisant de ces objets dans leurs lieux de conservation a entraîné trop souvent, depuis un siècle et demi, la destruction de ces éléments végétaux qui faisaient sens. Ces derniers ne semblent pas avoir intéressé outre mesure les collectionneurs et les conservateurs de musée qui se sont plus focalisés sur la forme des objets. Les divers éléments végétaux ont trop souvent été perçus comme de simples décorations si bien

\begin{abstract}
Some Kanak clubs preserved in various museums have bunches of plants tied to the handle. The study carried out in different European museums shows that the fronds of a particular variety of fern are most frequently used in these assemblages. The question posed is to know why this fern was used rather than another.

Keywords: fern, club, Kanak, New Caledonia, nonverbal message, fire, incendiary
\end{abstract}

qu'il existe très peu de documentation à leur sujet. C'est trop souvent la fonction matérielle de l'objet qui a été retenue plutôt que sa fonction immatérielle. La présence de tel ou tel végétal ligaturé sur un objet avait un sens mnémonique que tout homme initié se devait de comprendre, même au-delà de sa propre aire linguistique. Cependant, grâce à l'attention portée dans divers musées européens par les conservateurs, certains casse-têtes avec leurs végétaux ont pu être préservés dans leur intégralité. Nous étudions dans cet article des exemples montrant la présence récurrente d'un nombre restreint d'espèces végétales et, particulièrement, des fougères. Nous verrons que, parmi les très nombreuses espèces de fougères existantes localement, les Kanak en ont sélectionné seulement une ou deux pour ce type d'utilisation. Nous nous risquerons à faire

* Maître de conférences du MNHN et chargé de mission au musée du quai Branly, coiffier@mnhn.fr 
quelques hypothèses concernant la réalité d'une sorte de langage de signes codés entre des populations qui ne parlaient pas toujours la même langue. L'étude systématique de ces végétaux n’a, semble-t-il, jamais été entreprise et il est vraisemblablement trop tard pour comprendre leurs véritables significations.

\section{Les casse-têtes kanak et leurs éléments associés}

La fronde était l'arme préférée des Kanak avec la sagaie lancée à l'aide d'un propulseur, dont l'une des premières représentations européennes est un dessin de Nicolas Piron, dessinateur du voyage de Bruni d'Entrecasteaux (1791-1794), qui présente un guerrier avec son propulseur dans la main droite et un casse-tête étoilé dans la main gauche (La Billardière, 1799: $\mathrm{n}^{\circ} 35$ ). Les représentations photographiques du XIx ${ }^{\mathrm{e}}$ siècle montrent des guerriers kanak avec une lance dans la main gauche et un casse-tête dans la droite. À notre connaissance, aucun des premiers casse-têtes rapportés en Europe par la seconde expédition du capitaine Cook dans le Pacifique ne présente des végétaux associés. Il en est de même pour les casse-têtes rapportés quelques années plus tard par l'expédition de Bruni d'Entrecasteaux. Mais cela ne présuppose rien de leur présence, puisqu'ils ont pu être arrachés ou détruits avec le temps. Les collections ethnographiques françaises et étrangères ont vu ensuite affluer quantité d'armes kanak de combat rapproché comme les casse-têtes qui servaient à rompre les vertèbres du cou ou à défoncer le crâne ou le sternum de l'adversaire. Nombreuses parmi celles-ci ont été confisquées ou interdites d'usage dans certaines régions de la Nouvelle-Calédonie à la fin du XIx ${ }^{e}$ siècle. Elles furent remplacées quelques années plus tard par des fusils. Les casse-têtes ont très vite attiré l'intérêt des collectionneurs d'objets exotiques. Ces armes étaient en général fabriquées dans du bois extrêmement dur comme le gaïac (Acacia spirorbis Labill.) ou l'arbre de fer (Casuarina equisetifolia L.). Leenhardt (1932 : 159) évoque dans la légende de "La vierge de Nékliai " un casse-tête (koé are ou kwèe aare en ajïe, "racine-niaouli ») fait avec une racine de niaouli, Melaleuca quinquenervia [Cav.] S.T. Blake). Il existait des casse-têtes de formes différentes dont Fritz Sarasin (2009: 172-177) a décrit les principales caractéristiques pour les classer suivant cette nomenclature: casse-têtes droits à tête ronde, aplatie, en étoile ou en demi-boule, avec cannelures, casse-têtes à tête en forme de gland ou de champignon (droits ou recourbés), casse-têtes tête de tortue (ou tête d'oiseau) et casse-têtes en faucille. Un casse-tête rapporté par l'expédition de Bruni d'Entrecasteaux présente deux pointes opposées (Duuren et Mostert, $2007: 54)$.

Comme d'autres instruments et, particulièrement, les haches ostensoirs, certains casse-têtes avaient leur manche enveloppé, tout ou partie, dans des étoffes d'écorces (tapa) ou dans des fibres infra-pétiolaires de palmier ligaturées selon différentes techniques. Le liber utilisé pour fabriquer ces tapa, appelé autrefois balassor en Nouvelle-Calédonie, de couleur brunâtre pour celui issu du banian (Ficus prolixa Forst.) ou de couleur blanchâtre pour celui provenant du mûrier (Broussonetia papyrifera L.), avait une signification particulière. Ainsi, le tapa blanc était utilisé pour la confection des étuis péniens et des turbans. Ce gainage pouvait être réalisé sur tout le manche du casse-tête ou seulement à son extrémité. Il était fixé à l'aide de ligatures en cordelettes de poils de roussette teintes en rouge ou en fibres de cocotier (Sarasin, 2009 : 110-111). Ces ligatures étaient soit croisées de manière à former des losanges, soit enroulées en spirale. Dans son ouvrage sur la sorcellerie, Éliane Métais (1967: 168-169) note à la suite de Maurice Leenhardt (1930 : 234) l'importance des mots formés avec un terme désignant l'action de lier. D'après Isabelle Leblic :

« en paicî, on dit pi-pwö "nouer un signe (herbe, morceau de tissu), un tabou dans un endroit sacré" (Rivière, 1983 : 187), attacher quelque chose sur un bois tabou pour faire des invocations de pwö, "présent aux utérins, part des utérins" (ibid. : 212) et pii, “attacher, amarrer" (ibid. : 187) que l'on trouve aussi dans pinyî, nouer, nœud fait sur une perche sacrée dans lequel est concentrée toute la force donnée par l'oncle utérin à son neveu. " (Leblic, 2010 et com. pers.)

Parfois une bague en vannerie fine, agrémentée de bouclettes tressées réalisées en fibres végétales, était placée au milieu ou à l'extrémité de ce manche. Des amulettes renfermant des végétaux protecteurs pouvaient y être suspendues par des tresses en poils de roussette ${ }^{1}$ (Vieillard et Deplanche, 1863: 26 et 51). Des bouquets de feuillages pouvaient également être fixés parfois sur certains exemplaires ${ }^{2}$. Sarasin donne une description précise de ces casse-têtes :

«Le manche, comme d'ailleurs l'extrémité inférieure de la hampe, est souvent recouvert d'étoffe de liber ou de tissu, délicatement recouvert de cordons de coco qui se croisent. On utilise souvent aussi comme décoration des cordons de poil de roussette; il est habituel également de revêtir la hampe de feuilles de fougères ou de lycopodes. " (Sarasin, $2009: 172$ )

1. La roussette ou renard volant (Pteropus sp.) est une espèce de grande chauve-souris très commune en Nouvelle-Calédonie.

2. Cette coutume existait également au Vanuatu comme le montrent des cartes postales anciennes. 
Après les débuts de la colonisation, les enveloppements en tapa ou en tissus infra-pétiolaire de palmier ont été remplacés par des cotonnades européennes d'importation de couleurs rouge, bleue et parfois beige ou noire. À la fin du XIX ${ }^{e}$ siècle, les casse-têtes sont devenus rares et très recherchés par les Européens prêts à les acheter très cher (Lambert, 1976: 172), si bien que les bagnards se sont mis à en fabriquer pour les vendre aux voyageurs de passage à Nouméa (Vincent, 1895 : 84), mais ces pièces sont aujourd'hui aisément reconnaissables par les spécialistes.

Les photographies anciennes permettent de visualiser ces armes dans leur contexte de l'époque, mais très peu, comme celles d'Allan Hughan, présentent des hommes kanak portant des casse-têtes avec les végétaux ligaturés. Celle prise à Houaillou, en 1872, montre le grand chef Mindia Néja armé d'un casse-tête bec d'oiseau dans sa main droite (Leenhardt, 1930 : pl. xxxv, 1 ; Boulay, 1993 : 17 ; Brianchon, 2007 : 102). Des végétaux dépassant de l'enveloppement du manche sont nettement visibles. Deux photographies de Fritz Sarasin, l'une de face (Kasarhérou, 1993 : 40) et l'autre de profil (Sarasin, 2009 : fig. 1, pl. 65) présentent un porteur de masque tenant dans sa main gauche un casse-tête phallique sur le manche duquel est ligaturé un bouquet de fougères. Une photo, publiée dans le catalogue de l'exposition au musée des Beaux-arts de Chartres (2009 : 152), présente un homme de Moindou portant sur son épaule droite une hache métallique sur le manche de laquelle sont ligaturés des végétaux. Si l'on tient compte de la technique d'utilisation des casse-têtes, comme elle apparaît sur deux photographies ${ }^{3}$ d'une guerre entre les Poyes et les Touhos, où l'on voit nettement un homme assener un coup de casse-tête dans le dos d'un adversaire (Leenhardt, 1930 : pl. Ix et pp. 39-43), il semblerait que la bonne saisie de ces armes aient pu être gênée dans le cas où des fougères (ou autres végétaux) y étaient ligaturées. Mais certains casse-têtes avaient des fonctions très particulières et, pour cette raison, ne pouvaient être utilisés que par une certaine catégorie d'hommes.

"Ceux de Tegô s'affirment gardes du corps, guerriers au service du grand chef dont ils auraient reçu le bulaivi, le casse-tête en bec d'oiseau avec lequel ils doivent rythmer la danse au pilou et éventuellement en annoncer l'arrêt. Ce casse-tête appartient au grand chef pour le principe, mais seuls les Dambwe Hwiyanu peuvent le tenir en mains, et il ne doit jamais être utilisé pour tuer quelqu'un. "(Guiart, 1987 : 71)
Des dessins d'un ancien colon calédonien, $M$. Millet, publiés par Leenhardt (1930 : 166-167), montrent, lors d'une cérémonie de présentation d'un nouveau chef, des femmes mariées du clan maternel de ce dernier, allant au-devant des paternels en arborant, comme des hommes, des sagaies et en brandissant dans leur main droite des casse-têtes à longues pointes ${ }^{4}$ dont plusieurs présentent des enveloppements enserrant pour l'un d'entre eux des végétaux bien visibles. Ces femmes dans une danse virile, célébrant leur neveu devenu chef de tribu, allaient arracher les ornements (aigrette, plumes, chapeaux) des hommes du groupe opposé qui devaient rester dignes. Ce rituel évoque celui des femmes iatmul de Nouvelle-Guinée travesties en hommes qui, durant certains naven honorant également un neveu héros, pouvaient avoir un comportement tout à fait similaire, particulièrement, jadis, lorsque ce neveu avait accompli son premier meurtre (Bateson, $1971: 22-23$ et 29).

Force est de constater que la destruction volontaire ou involontaire des végétaux associés à certains casse-têtes a fait disparaitre une partie de leur histoire. Les provenances des casse-têtes conservés dans les musées ou chez les collectionneurs n'étant pas toujours connues, il s'avère donc maintenant difficile de tirer des conclusions relatives à des pratiques régionales particulièrement significatives selon les zones culturelles et linguistiques kanak.

\section{Les casse-têtes associés à des plantes, dans les musées français ${ }^{5}$ et européens}

Nous nous intéressons ici principalement aux casse-têtes sur lesquels se trouvent ligaturées des plantes diverses, sachant que ceux dont le manche se trouve seulement enveloppé de fibres infrapétiolaires de palmier sont relativement nombreux dans les collections (МQв. 71.1903.6.4, $71.1937 .10 .3,72.1955 .1 .2)$. Un dessin de couverture du menu du 46 dîner Gabriel de Mortillet montre un casse-tête bec d'oiseau sur lequel est ficelé un gros bouquet. Cela montre l'intérêt porté à cette pratique par certains membres de l'École d'Anthropologie de Paris au début du $\mathrm{Xx}^{\mathrm{e}}$ siècle (Musée des Beaux-arts de Chartres, 2009 : 31). Les photos des panoplies d'armes kanak présentées sur les murs du musée d'ethnographie du Trocadéro permettent d'y distinguer plusieurs casse-têtes de forme phallique envelop-

3. Ces photographies de combats guerriers représentaient des mises en scène destinées au marché de la carte postale.

4. Sarasin $(2009: 201,205$ et pl. 61,4 et 5) appelle ces accessoires des verticilles de danse et s'interroge sur leur signification.

5. Nous adressons tous nos remerciements à l'UnEsCo pour la subvention accordée au professeur Jean Guiart et destinée à compléter le répertoire des collections océaniennes des musées de province en France. En 2002, alors que nous étions chargés des collections océaniennes au musée de l'Homme, nous avons pu bénéficier d'un reliquat de cette subvention, gérée par la Société des Amis du musée de l'Homme et nous l'avons utilisé pour répertorier dans une vingtaine de musées divers objets océaniens dont les casse-têtes kanak associés à des végétaux. 


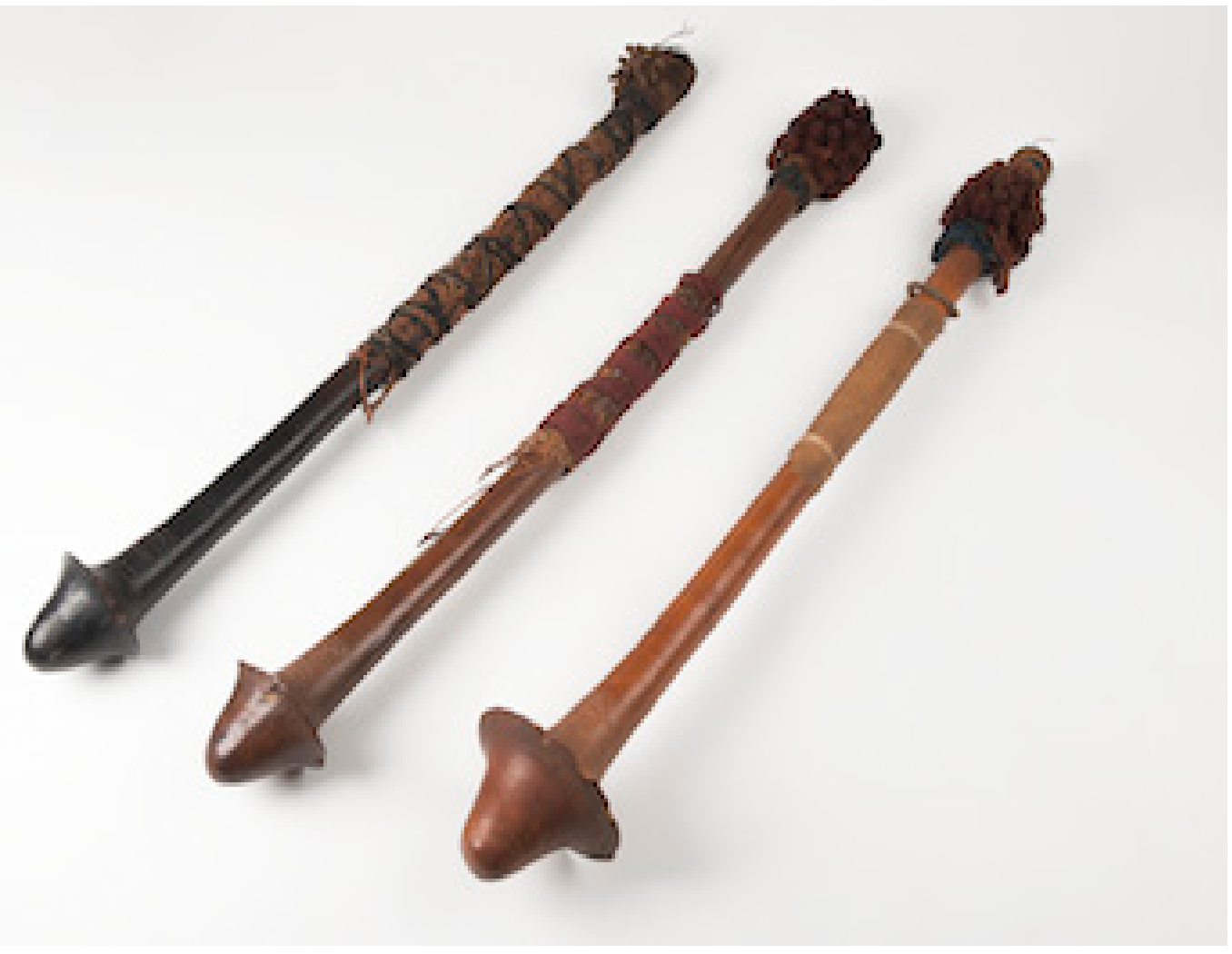

Рното 1.- Trois casse-têtes du musée des Confluences à Lyon (Inv. FR-LYN. 60000714, FR-LYN. 60003457 et FR-LYN. 2012.0.32) avec leur manche enveloppé de frondes de fougère (C) Département du Rhônemusée des Confluences, cliché de Patrick Ageneau, 2013)

pés de végétaux (Luquet, 1926 : pl. x, 4 ; Degli et Mauze, $2000: 57)$. Il est permis de s'étonner que ces végétaux aient pu résister aux mauvaises conditions de conservation (différence de températures, dessiccation, humidité, insectes...) dans cet ancien musée parisien et aux nombreuses manipulations lors de leurs déplacements. La majorité de ces casse-têtes se trouve maintenant dans les réserves du musée du quai Branly qui en possède ainsi au moins cinq de forme phallique avec des végétaux ligaturés. L'un d'entre eux (MQB. 71.1882.20.3, don de Mme Vimont) présente des feuilles de fougère (vraisemblablement Pteridium esculentum) enveloppées dans une petite vannerie. Trois exemplaires, provenant du don Ardouin, sont intéressants par la diversité de leurs végétaux. Le premier (МQв. 71.1893.16.10) est associé à une branchette de gaïac (Acacia spirorbis) avec ses feuilles ligaturées par une botte de graminées (Imperata cylindrica); le second (МQв. 71.1893.16.11) présente une fronde de fougère (Pteridium esculentum) associée à des rameaux de gaïac (Acacia spirorbis) et de salsepareille (Smilax sp.) également ligaturés avec des herbes (Imperata cylindrica) et un tissu européen; des frondes de fougère (Pteridium esculentum) sont fixées à l'aide d'un tissu européen sur le troisième (МQв. 71.1893.16.12). Par contre, il ne reste que quelques brindilles non identifiables ligaturées à l'aide d'un tissu européen sur un casse-tête du don d'Octave Opigez (MQв. 71.1893.20.5). Un cassetête en bec d'oiseau (MQB. 71.1885.52.12.D), provenant du musée d'archéologie national, présente un manche enveloppé d'une cotonnade imprimée rouge (style Liberty) avec des motifs végétaux blancs ligaturée avec un tissu bleu à points blancs. Doit-on imaginer que le propriétaire avait estimé que ces tissus pouvaient remplacer de réels végétaux?

Il semblerait que les végétaux ligaturés sur les casse-têtes aient été mieux conservés dans les musées de province, du fait de manipulations moins fréquentes dans les réserves ou dans des vitrines. Le muséum d'histoire naturelle de La Rochelle conserve ainsi deux casse-têtes bec d'oiseau provenant de la collection du docteur Loppé. Sur l'un d'eux (Inv. H.4333), dont le manche est enveloppé de tissus européens rouges, se trouve liées quelques frondes de fougère. Le second (Inv. H.4233), dont le manche est enveloppé de tissu européen de couleur blanc et bleu, est ligaturé avec des fibres de palmier. Le musée breton de Quimper conserve un casse-tête avec l'inscription don de l'archiviste R.F. Le Men vers 1880. La fiche d'objet indique :

«On y attachait un bouquet de végétaux dont le pouvoir renforçait l'efficacité de l'arme. " 


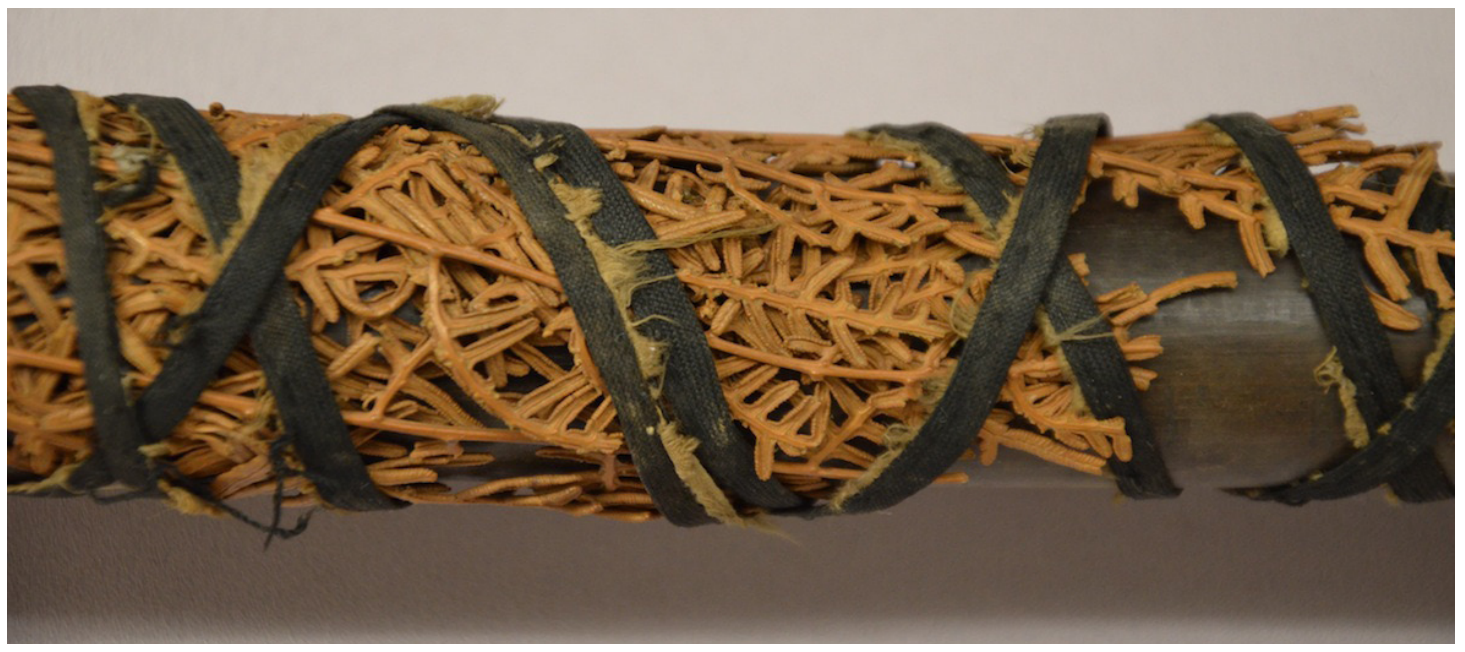

Рното 2. - Gros plan des frondes de fougère Pteridium esculentum ligaturées sur le manche du casse-tête Inv. FR-LYN. 60003457 du musée des Confluences à Lyon (C) Inventaire du patrimoine kanak dispersé, Paris, 2012)

Le musée des Confluences de Lyon conserve également trois casse-têtes provenant du don Dumont en juin 1880. Ces derniers présentent tous les trois des bouquets de frondes de fougère Pteridium esculentum ligaturées sur leur manche (Inv. FR-LYN. 60000714, FR-LYN. 60003457 et FR-LYN. 2012.0.32), mais l'un d'entre eux est également enveloppé dans de la fibre infrapétiolaire de palmier (voir photos 1 et 2).

Le muséum d'histoire naturelle de Toulouse conserve deux casse-têtes de forme phallique (Inv. MNHT.ETH.AC.NC.32 et MNHT. ETH.AC.NC.39) et deux casse-têtes bec d'oiseau (Inv. MNHT.ETH.AC.NC.6 et MNHT. ETH.AC.NC.13, voir photo 3) tous les quatre associés à des fougères (Pteridium esculentum).

Dans sa classification des casse-têtes, Sarasin (2009 : 172-177) évoque des spécimens conservés dans les musées européens (Bâle, Berlin, Cambridge, Munich, Rome, Stuttgart, Vienne) et donne la description d'exemplaires du musée d'ethnographie de Bâle. Il signale qu'il est habituel de revêtir la hampe avec des feuilles de fougère et de lycopode, ce dernier végétal n'étant cependant signalé que sur un casse-tête du musée de Bâle ( $c f$. infra) et sur des coiffures cylindriques en vannerie (Sarasin, 2009 : 157). Il est fort probable que, vu sa fragilité, ce matériau ne se soit pas conservé correctement ${ }^{6}$.

"À la hampe de celui de Hienghène [...], au très long gland à un bord tranchant, de $7 \mathrm{~cm}$ de diamètre, est attachée une botte de feuilles de fougère et d'herbe. [...] Le casse-tête de Paimboa [...], brun rouge sombre, magnifiquement poli, est revêtu sur presque toute sa longueur de feuilles de fougères Glei- chenia, tenues par une bande bleue et de l'étoffe de liber enroulée ; le diamètre de son petit gland n'est que de $5 \mathrm{~cm}$. [...] Le casse-tête brun clair de la vallée de Négropo [...], fortement coudé [...], a le manche enveloppé d'étoffe et de cordons de coco ; une botte de lycopodes est attachée à la hampe avec des chiffons européens. » (Sarasin, 2009 : 174)

Outre le musée de Bâle en Suisse, celui de SaintImier possède également un casse-tête phallique avec des frondes de Pteridium esculentum (MSI HUM.14, voir photos 4-5).

Les végétaux ligaturés se trouveraient donc seulement sur deux types de casse-têtes, sur ceux en bec d'oiseau et, plus fréquemment, sur ceux de forme phallique. Sarasin (2009 : 205 et pl. 53, 7) évoque également l'utilisation de bouquets végétaux sur des palettes de danse dont il attribue la forme à une imitation des haches de métal européennes. Des photographies de deux d'entre elles présentent un manche agrémenté de bouquet (ibid., 2009: pl. 61, 1 et 2), Sarasin précise que celle du musée de Bâle provenant du village de Marengu (région de Canala) est couverte de fougère Gleichenia sous une bande de tissus. Des casse-têtes de différentes formes ou des hommes porteurs de ces armes ont souvent été représentés sur les bambous gravés; par contre, il ne semble pas, à notre connaissance, y avoir de telles représentations avec des végétaux ligaturés. Dans ses interprétations des bambous gravés. Marguerite Lobsiger-Dellenbach (1967: 68, 72-73) évoque à plusieurs reprises des motifs représentant des fougères et elle qualifie le créateur présumé de deux d'entre eux (MQB.72.1962.2.5 et MQB.72.1962.2.6) de "Maître à la fougère ".

6. Sur les seize espèces de lycopode recensées en Nouvelle-Calédonie, neuf sont communes (cinq épiphytes et quatre terrestres). Lycopodium cernuum est utilisé pour garnir les bouquets de fleurs et Lycopodium phlegmaria pour se parer (Sarasin, 2009 : 157). N.B. Quinze espèces sont actuellement acceptées par les botanistes. 


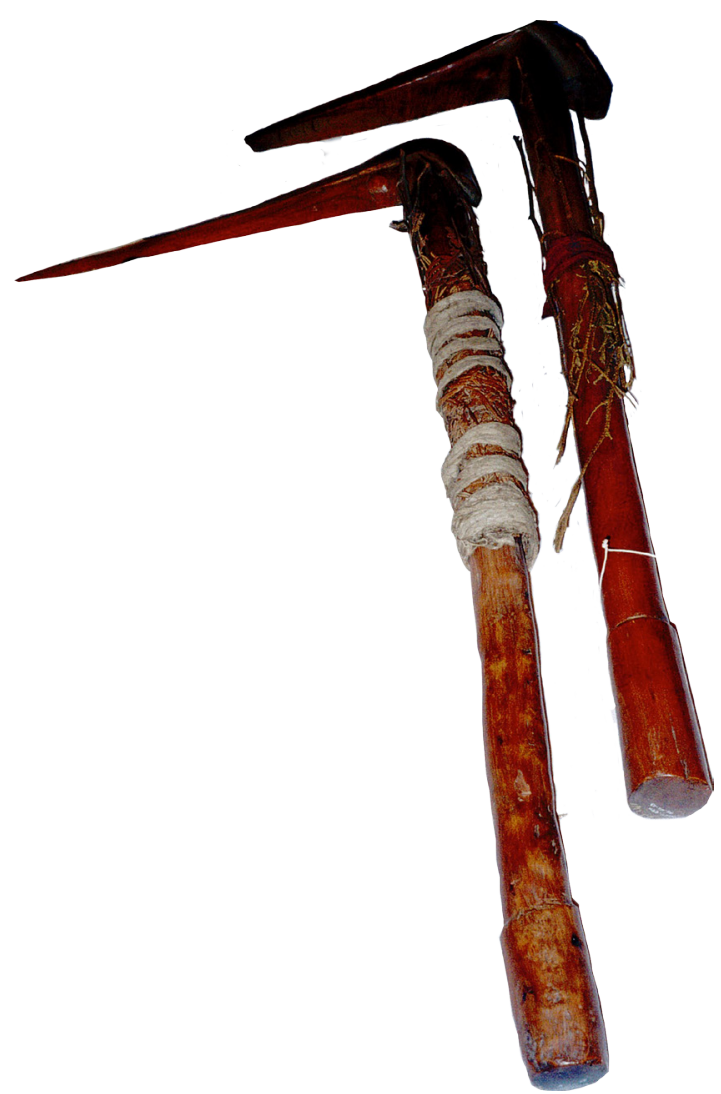

Рното 3. - Deux casse-têtes bec d'oiseau (a-b) du muséum de Toulouse (Inv. MNHT.ETH. AC.NC.6 et MNHT.ETH.AC.NC.13) avec leur manche enveloppé de frondes de fougère Pteridium esculentum (cliché de l'auteur, 2002, avec l'aimable autorisation de Frédérique Gaillard, photothèque du Muséum de Toulouse)

Sur l'un d'eux est représenté un couple de lézards au milieu de casse-têtes bec d'oiseau et d'une étrange végétation rappelant des frondes de lycopode ou de certaines variétés de fougère. Le $\mathrm{Mu}-$ seum für Völkerkunde de Vienne conserve un bambou gravé (inv. n007666, coll. Goldbeck) sur lequel se trouvent ligaturés des fougères (Pteridium esculentum) et des propulseurs en fibres végétales à l'aide d'herbes (Imperata sp.) et de tissus européens (E. Kasarhérou, com. pers.). C'est principalement deux espèces de fougère ligaturées que l'on trouve ainsi pour un corpus relativement restreint d'une vingtaine de pièces citées ci-dessus. Ce qui est néanmoins suffisant pour en tirer quelques conclusions. Les espèces de fougère sèches peuvent être identifiées grâce à l'architecture de leur fronde, à la forme de leurs pinnules ${ }^{7}$ et à l'implantation de leurs sporanges.

\section{Divers usages et représentations des fougères chez les Kanak}

Les fougères arborescentes semblent avoir été perçues par les Kanak comme une catégorie de plantes différente quoique tout aussi importante que les fougères herbacées.

"Dans certaines régions du pays kanak, le nom de la fougère arborescente signifie le commencement du pays des hommes car elle évoque les origines. Plusieurs clans considèrent que l'Ancêtre est sorti de son tronc creux provoquant leur apparition au monde» (Kasarhérou et Wedoye, 1998 : 10)

Leur bois était utilisé pour la fabrication des pointes de sagaies et leurs bourgeons comme contraceptifs (ibid., $1998: 10-11$ ). Vieillard et Deplanche (2005: 91-92) sont très précis sur les divers usages locaux des fougères comme l'arborescente Cyathea vieillardi dont les Kanak récoltent par incisions dans le stipe, ou à la base des frondes, un suc mucilagineux qui se coagule en une sorte de gelée assez fade et peu nourrissante.

"Les rhizomes des Gleichenia dichotoma et G. flabellaris ${ }^{8}$ sont également utilisés comme alimentaires dans les années de disette [...]. De toutes les fougères comestibles, la plus précieuses et la plus recherchée, à cause de la grande quantité de matières nutritives qu'elle renferme est l'Angiopteris evecta Hoff. [...] Les jeunes frondes de l'Helminthostachys zeylanica Hook. peuvent être préparées et servies en guise d'Asperges [...] Les rhizomes durs et amers de Pteris esculenta Forst. sont peu prisés ${ }^{9}$; ils ne sont guère employés que dans les cas extrêmes [...] Broyées et triturées avec de l'huile de coco, les pinnules aromatiques du Polypodium phymatodes Linn. et de l'Angiopteris evec$t a$, font la base d'un liniment très employé par la médecine indigène contre les douleurs rhumatismales. " (Vieillard et Deplanche, 2005 : 91-92)

Fritz Sarasin (2009 : 79) signale quelques autres espèces comestibles comme l'Alsophila sp. ou la

7. Les pinnules d'une fronde de fougère correspondent aux folioles d'une feuille composée.

8. Sur quatre espèces de Gleicheniaceae présentes en Nouvelle-Calédonie, deux sont endémiques (Sarasin, 2009 : 79). D'après Florical (http://www.botanique.nc/herbier/florical) consulté le 19/12/2012, on connaît actuellement six espèces de Gleicheniaceae dont une seule du genre Gleichenia en Nouvelle-Calédonie, la Gleichenia dicarpa R. Br., les autres sont Dicranopteris linearis (Burm. F.) Underw, Sticherus brackenridgei (E. Fourn.) H. St John, Sticherus flabellatus (R. Br.) H. St John, Sticherus montaguei (Compton) Nakai et Stromatopteris moniliformis Mett (com. pers. de Pierre Cabalion).

9. La toxicité de cette espèce, bien documentée dans la littérature, est notamment due au ptaquiloside, présent par exemple dans les frondes, provoquant chez les herbivores des cancers au niveau de l'iléon et de la vessie (Smith et al., 1988). Cette molécule mutagène provoque aussi des leucopénies et des syndromes hémorragiques diffus. La plante est pourtant considérée comme une ressource alimentaire humaine traditionnelle dans divers pays (Nouvelle-Calédonie, Nouvelle-Zélande, Japon), mais souvent après cuisson (Pierre Cabalion, com. pers.). 


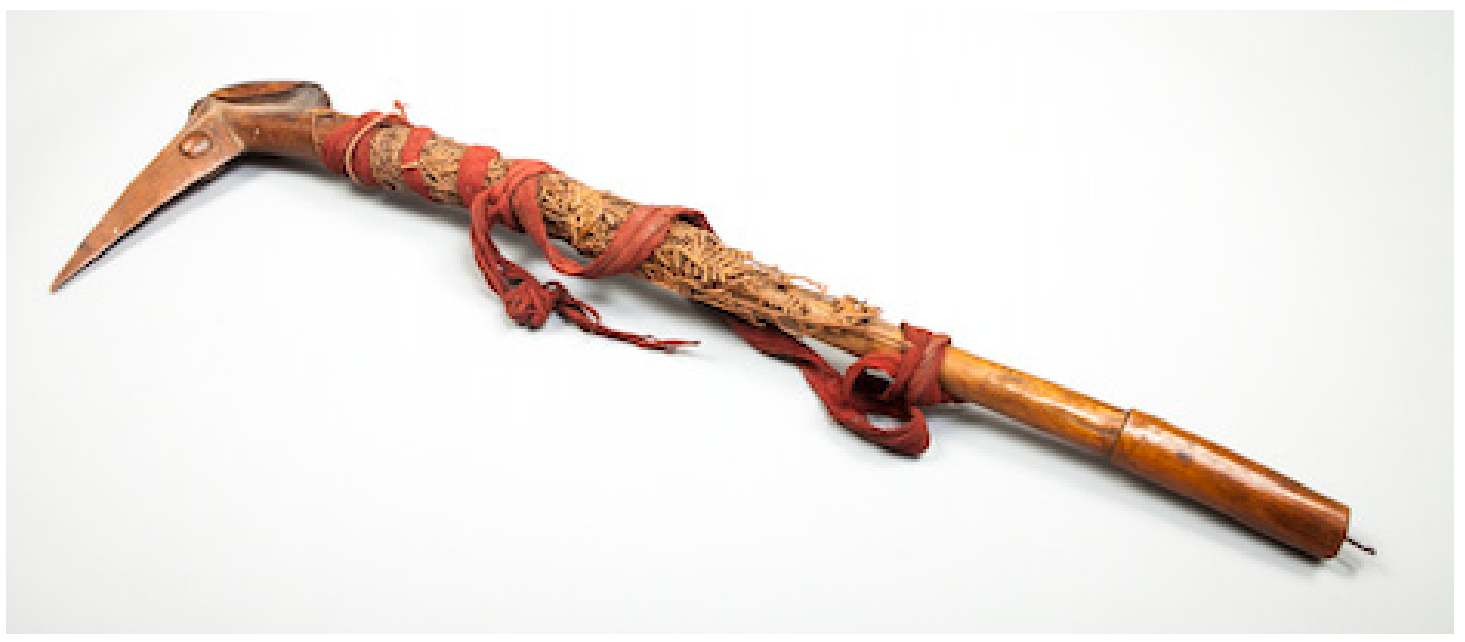

Рното 4. - Casse-tête du musée de Saint-Imier (Inv. MSI HUM.14) avec son manche enveloppé de frondes de fougère (C) musée de Saint-Imier, Suisse, cliché de J.-M. Künzi, 2013)

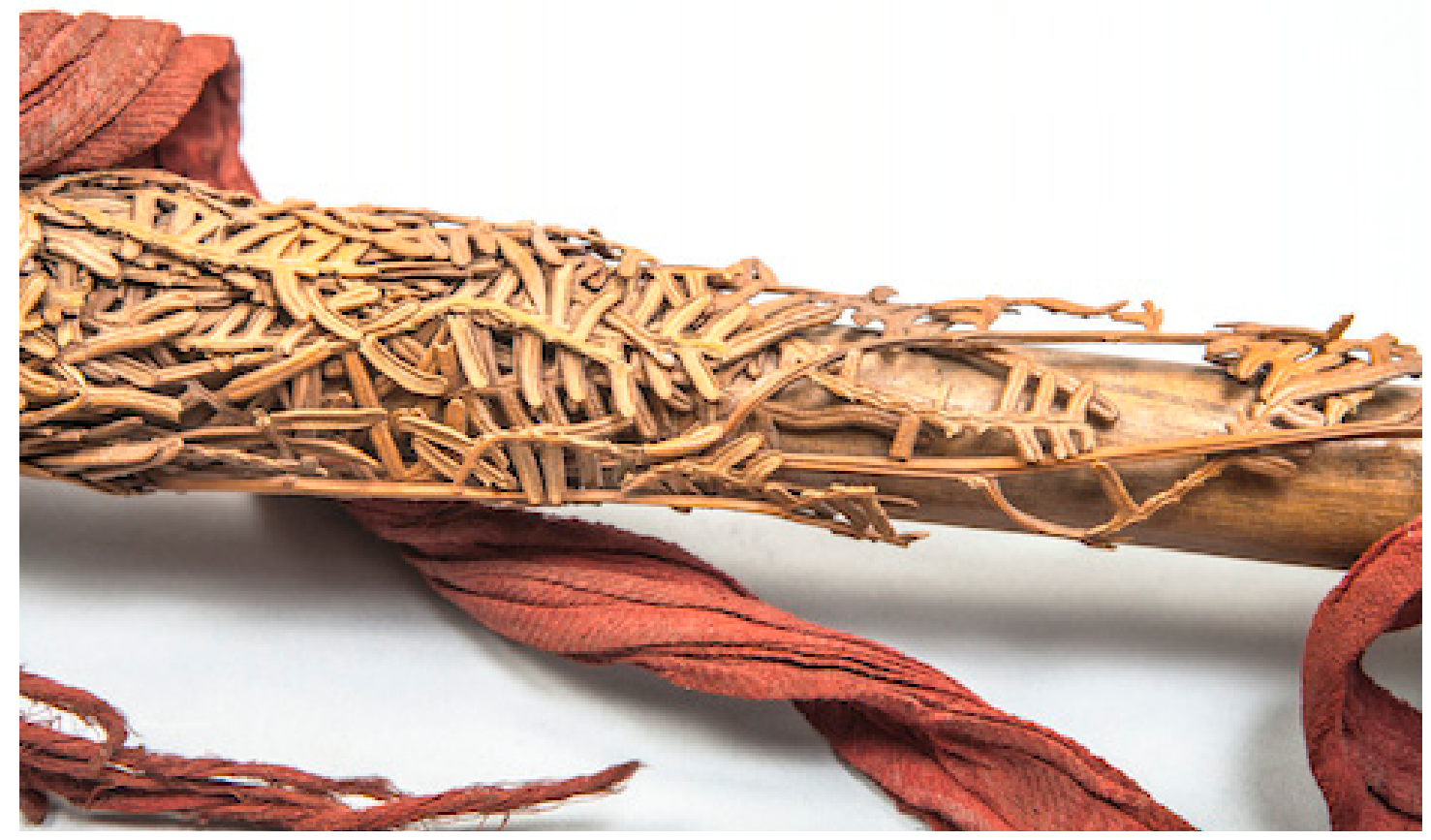

Рното 5. - Gros plan des frondes de fougère Pteridium esculentum ligaturées sur le manche du casse-tête Inv. MSI HUM.14 (@) musée de Saint-Imier, Suisse, cliché de J.-M. Künzi, 2013)

Marattia sp. dont la chair rougeâtre de la base des frondes a le goût désagréable de mauvaise carotte. Selon Vieillard et Deplanche (2005 : 92), les longues radicelles noires et brillantes du Blechnum gibbum et de Lomaria sp. Willd. servaient dans le nord de l'île à orner le sommet des cases ou les perruques de certains masques. Cette information est reprise par Sarasin (2009: 157). Guiart (1987: 129) et Kasarhérou (1990: 147, 1993 : 31-33) la complètent en faisant référence à une légende du clan Wéoumé (Wêxumé), rapportée par Maurice Leenhardt, où un enfant fabrique deux masques, l'un avec une cotte de plumes et l'autre avec une cotte en feuilles de fougère :
«Dans la région de Houaïlou, il existait des masques dont les manteaux étaient faits des feuilles de la petite fougère (Blechnum sp.) qui pousse au bord des ruisseaux, dite wèri en langue ajië. Le masque était alors appelé pûrûu-wèri, mot-à-mot plume/poil de Blechnum sp. " (Kasarhérou, 1993 : 38-39)

Kasarhérou (1993 : 38) signale également que des racines de fougère étaient utilisées dans les régions centre et sud de la Nouvelle-Calédonie pour constituer la barbe de certains masques, comme celui conservé au musée territorial de Nouvelle-Calédonie à Nouméa (Inv. MNC 86.5.198). Cette fougère wèri avait également divers usages médicaux, elle pouvait être frois- 


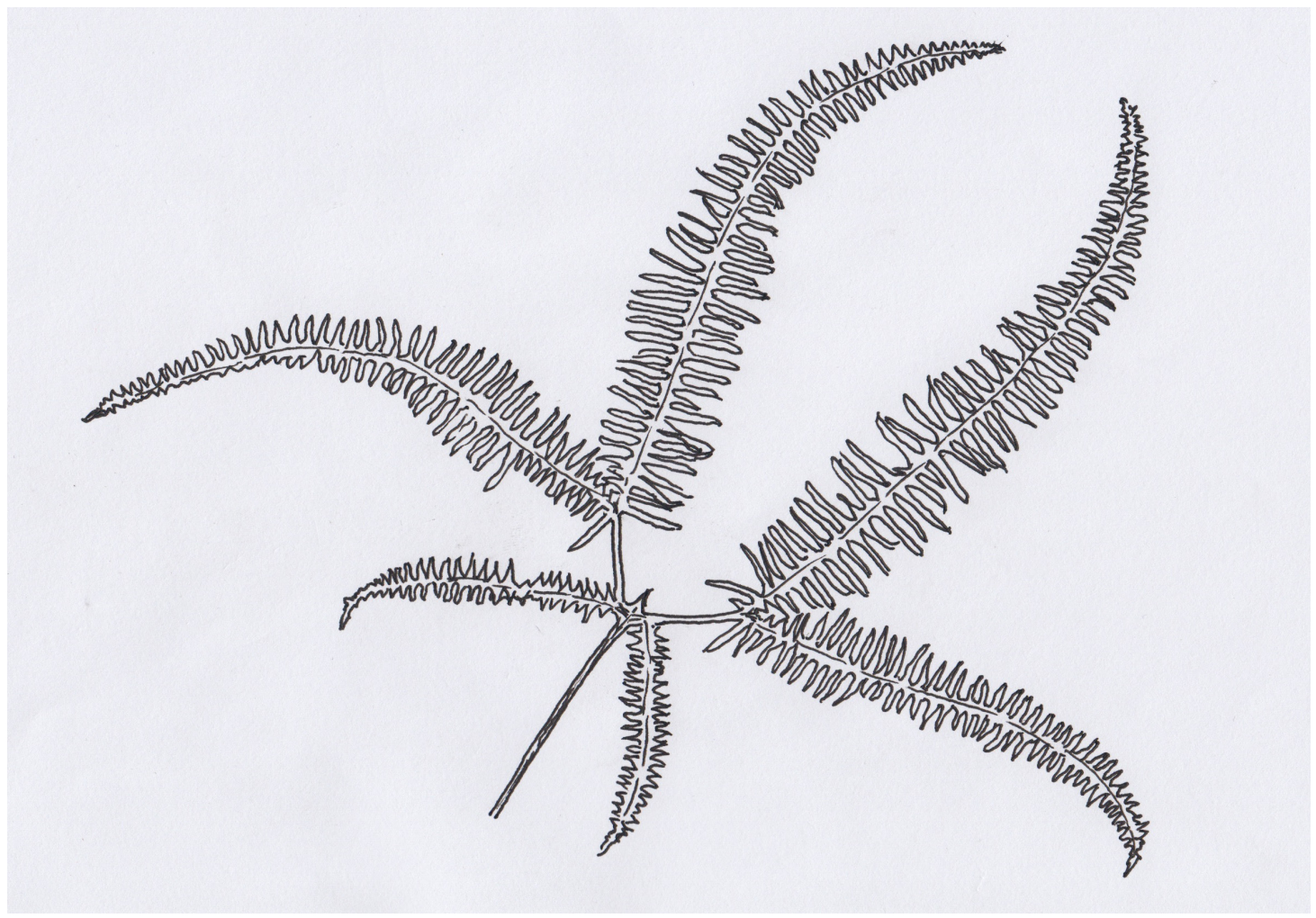

Croquis 1. - Une fronde de fougère Dicranopteris linearis (Burm. F.) Underw. montrant sa division binaire si caractéristique, d'après Suprin, $2011: 79$ (dessin de l'auteur, 2013)

sée, écrasée et crachotée dans les oreilles et sur le corps d'un malade. Les poils de ses racines étaient roulés en torche pour enfumer le corps de celui-ci. Selon Leenhardt (1935: 354) c'était un remède contre la folie provoquée par le totem du clan Erigoami, qui en conservait le secret. Les larges fougères Asplenium nidus croissant sur les arbres (Cronin, 1989: 126-127) étaient considérées comme les plumets de la coiffure de la divinité Gomawe et ce sont les frondes de ces fougères qui étaient utilisées pour combler les interstices entre le faittage et la corbeille de gaulettes de la toiture d'une case (Leenhardt, 1935 : 47). Selon Patouillet, cité par Sarasin (2009: 244), des frondes de fougères arborescentes associées à des pièces de tapa blanc (balassor) constituaient les énormes chapeaux des deuilleurs d'un mètre de diamètre. Les porteurs devaient les tenir avec leurs mains et ils étaient interdits à la vue des femmes. Divers auteurs anciens ont noté l'intérêt des Kanak pour les parures corporelles et particulièrement pour leur chevelure où les frondes de fougère avaient une place de choix (Luquet, 1926 : 4).

"Les frondes élégantes du Gleichenia dicarpa R. Br., du Lygodium reticulatum Schk., du Dicksonia thyrsopte- roides Mett., du Stromatopteris moniliformis Mett., des Lycopodium cernuum et mirabile sont généralement employés pour la confection des couronnes, dont les indigènes se parent les jours de fête. » (Vieillard et Deplanche, $2005: 92$ )

Les fougères Gleichenia sp. étaient encore utilisées, dans les années 1960, sous les nattes de vannerie pour constituer des " matelas à ressort " (Jacqueline de la Fontinelle, com. pers.). Selon le dictionnaire de Lercari (2002), les enfants utilisaient le rachis de la fronde de la fougère médicinale jué en langue ajië (Sticherus flabellatus ${ }^{10}$ tout en conservant son attache sommitale pour jouer à l'escrime de casse-tête (camù). Il est vrai que les jeunes frondes, avec leur tête enroulée en spirale, peuvent évoquer certains types de casse-têtes. Cette fougère est également utilisée pour un autre jeu consistant à accrocher deux jeunes tiges par leur attache sommitale et à tirer ; celle qui casse la première fait perdre celui qui la tient (Lercari, 2002). Selon le dictionnaire de langue ajië de Leenhardt (1935 : 170) le terme lù signifie nouer, attacher, natter de petits fils, tresser. Il s'emploie dans les ouvrages de natterie et de sparterie fine, comme celles des têtes de monnaie. Selon Emmanuel Kasarhérou (com. 


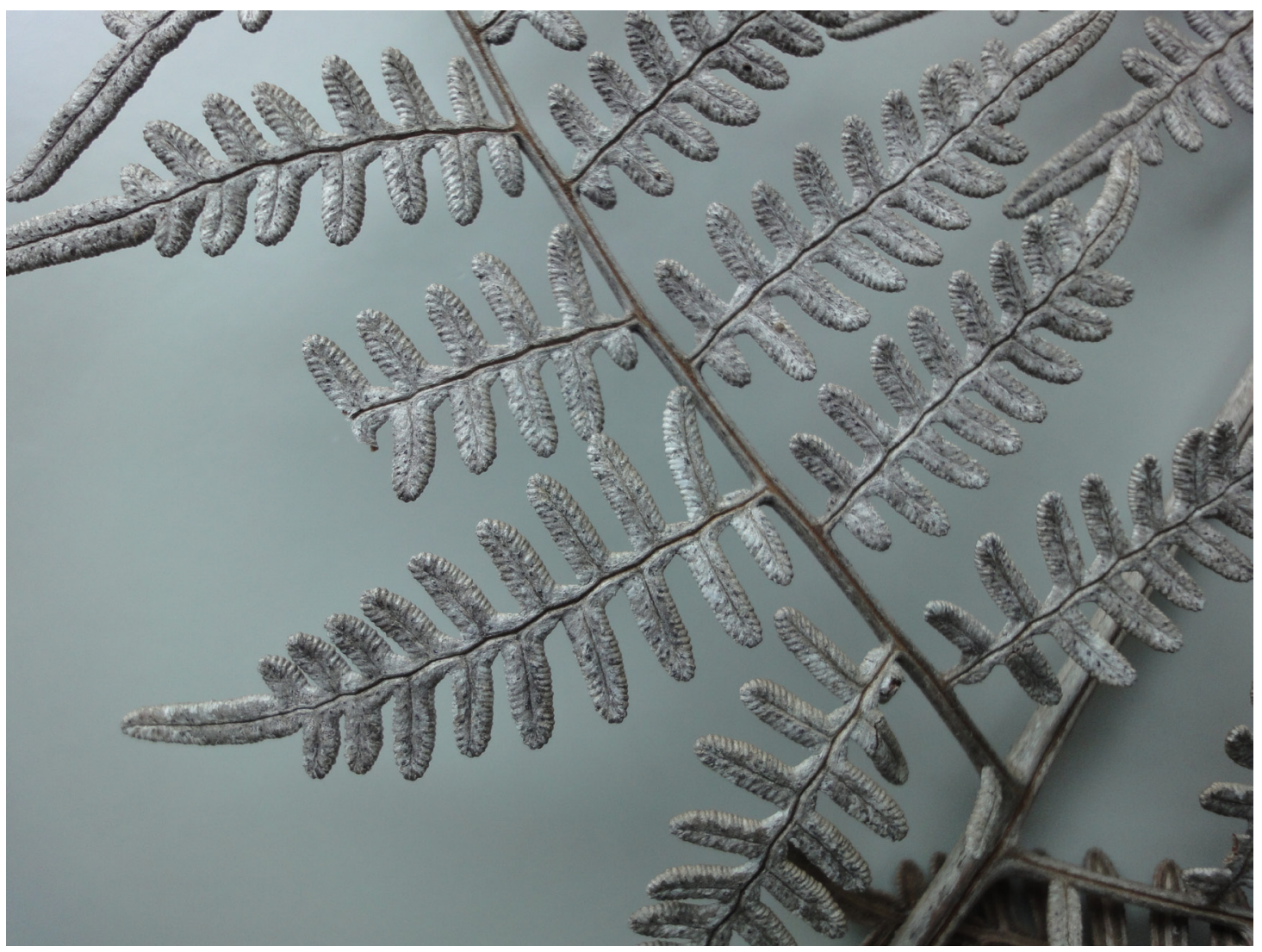

Рното 6. - Élément de fronde de Pteridium esculentum G. Forst. Cockayne (cliché de l’auteur, 2013)

pers.), la fougère jué porte également le même nom de lì en langue ajië. Si le mot lù désigne également l'herbe Apluda mutica L. (Lercari, 2002), il n'est donc pas exclu que des éclisses de rhizome de fougère jué (Sticherus flabellatus) aient été utilisées jadis, avant la colonisation, par les hommes pour la fabrication de vanneries très fines comme celles ornant les monnaies, les plaquettes de lances et les manches de casse-têtes.

Les Kanak divisent la végétation en deux catégories, les plantes humides (canne à sucre, bananes, taros) associé au monde féminin et les plantes sèches associées au monde masculin (igname, cordyline). Selon Emmanuel Kasarhérou, en ce qui concerne la région de Houaïlou, toutes les espèces de fougères n'appartiennent pas forcément à une même catégorie (Leenhardt, 1930 : 23). Chaque clan entretenait une relation intime avec un certain nombre d'espèces végétales et animales qui constituaient son entité totémique (Leenhardt, 1935 : 354). C'est ainsi que l'on pourrait penser que les végétaux ligaturés sur les manches de casse-tête auraient appartenus au totem du propriétaire de l'arme. Mais pour valider cette hypothèse, il faudrait connaître la région d'origine de chacun des casse-têtes en question et les totems d'origine des anciens propriétaires.
Le manque de diversité de ces végétaux ne permet guère de corroborer cette hypothèse. Cependant il apparaît que certaines espèces de fougère, ainsi que les lycopodes ${ }^{11}$, étaient associées au feu et aux incendies.

L'organisation des jardins et des zones de culture kanak étaient basées, jadis, sur une gestion équilibrée de l'eau, "l'humide ", et du feu, "le sec" (Leenhardt, 1930 : 23). Les Kanak pratiquaient la technique du brûlis pour préparer leurs jardins aux cultures. Cette utilisation du feu avait alors des vertus bénéfiques, mais parfois des conséquences inattendues. Certaines fougères comme la Gleichenia brackenridgei, qui apprécie les lisières de forêt, sont aisément inflammables lorsqu'elles sont sèches (Suprin, 2011: 80). C'est également le cas de la grande fougère aigle Pteridium esculentum et de la Dicranopteris linearis, une petite fougère très commune sur les sols schisteux et siliceux des contreforts de la chaine centrale où les feux de brousse sont fréquents. Elles sont parmi les premières plantes à repousser sur les terrains incendiés et leur croissance rapide étouffe toute autre végétation si bien qu'elles maintiennent ces terres sous leur seule domination (Suprin, 2011 : 79). 
«La fougère aigle se révèle très néfaste. Elle porte une lourde responsabilité dans la destruction des forêts du sud de la Grande Terre : elle forme avec le feu une dangereuse plante de couverture naturelle... ses rhizomes lui permettent de survivre aux incendies, tandis que ses frondes sèches, longues à se décomposer, s'offrent en feutrage dense dans le maquis et brûlent comme de l'étoupe. »(Suprin, 2011 : 75)

Il est avéré historiquement que les vainqueurs d'une guerre inter-villageoise brûlaient les cases de l'ennemi, coupaient les cocotiers et ravageaient ses plantations (Vieillard et Deplanche, 2005 : 48 ; Lambert, 1976 : 177).

"La fougère est une plante qui flambe facilement et favorise la marche rapide du feu. Si donc, la fougère domine dans le paquet [magique ...], c'est la dévastation du pays ennemi par l'incendie qu'on demande. " (Lambert, 1976 : 174)

"Une torche minuscule, et qui a été allumée et éteinte pour porter la trace de l'usage qu'on en fera, est serrée dans un nœud et expédiée à l’ennemi : “je viendrai brûler vos cases”." (Leenhardt, 1930 : 45)

Il apparaît donc que les fougères sèches, par leur faculté à s'enflammer facilement, étaient associées avec le feu dans les représentations de nombreuses sociétés kanak, mais en était-il de même pour les frondes de fougère fraîchement cueillies ? Dans ses recherches sur la maladie et les savoirs thérapeutiques kanak, Christine Salomon apporte quelques éléments pour comprendre les fondements de ces pratiques incendiaires :

«À la symptomatologie foudroyante des atteintes provoquées par les esprits des morts (les divinités) sur le modèle de la guerre d'extermination - correspondent des traitement d'éradication par le souffle et la flamme du feu, associés à la symbolique masculine et guerrière" [...]. L'action du soignant consiste à combattre la maladie, ce que la langue a'jië exprime par les expressions "défaire", "frapper la maladie", la langue paicî par "piétiner pour tuer", et encore par "lutter par le feu contre les esprits ancestraux" [...]. Enfin, on se souvient que ce sont la fumée d'une torche enflammée et le souffle qui chassent les esprits ancestraux d'un lieu (ou d'une personne). La technique qui associe à la force du feu celle du souffle, du vent, se nomme en a'jië "chasser par la fumée de la torche", en paicî "faire de la fumée contre", en encore "souffler le feu contre”. »(Salomon, $2000: 75,81$ et 144)

\section{Essai d'interprétation de la signification des végétaux fixés sur les casse-têtes}

Le père Lambert $(1900: 36-40)$ et le pasteur Leenhardt $(1930: 125,245)$ se sont particulièrement intéressés, en tant que prêtres, aux paquets magiques et aux amulettes végétales, dont certaines étaient fixées sur divers instruments, comme les casse-têtes et haches ostensoirs, ou

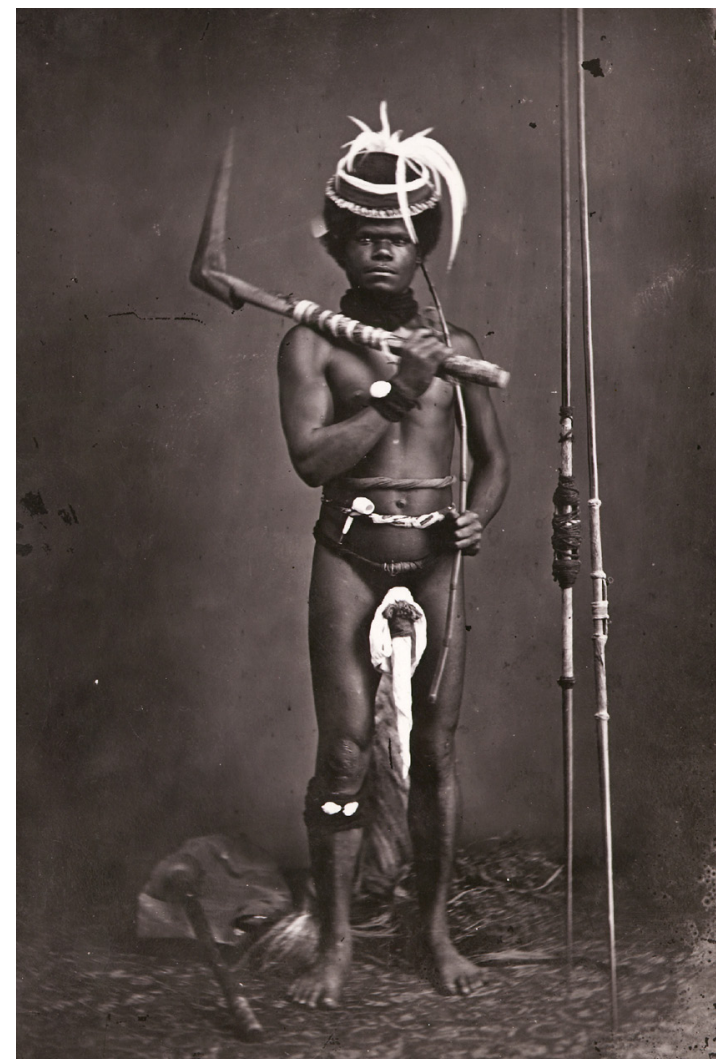

Рното 7. - Grand Chef Mindia Néja tenant dans main droite un casse-tête enveloppé de frondes de fougères (cliché d'Allan Hughan, Houaïlou, 1872, Archives de Nouvelle-Calédonie, Album Maurice et Raymond Leenhardt 2 Num 9 - 92)

placées à l'intérieur des bambous de voyage. Une opération consistait jadis à préparer un sortilège en utilisant parfois des paquets (Vieillard et Deplanche, 1863 : 28 ; Lambert, 1976 : 36-39). Elle était l'apanage, dans chaque village, d'une famille responsable des magies. Les ingrédients constituant ces paquets pouvaient être très divers, mais ce qui était primordial, c'étaient les paroles prononcées lors de leur préparation ou de leur utilisation.

"Les plantes, disent les vieux, n'ont pas de vertus propres; elles ne sont que le matériel symbolique sur lequel l'officiant prononce les paroles sacrées qui lui permettront de véhiculer la puissance de l'ancêtre. » (Tjibaou, $1976: 284$ )

Selon les régions, ces paquets étaient ficelés de différentes manières à l'aide de liens d'herbes (Lambert, 1976: 36-39). Le musée du quai Branly conserve un paquet magique donné par Maurice Leenhardt (MQB.71.1934.2.44) et constitué d'un morceau de tapa enveloppant une cordelette de poils de roussette, une pierre et des fragments de roseau, de fougère séchée et de filet. C'est vraisemblablement le même (ou un paquet similaire pour faire venir la pluie et 
dénommé bore) que l'on peut voir dans l'ouvrage de Leenhardt (1930: 124 et pl. XvII, 2) sur une photographie qui présente les différents éléments contenus à l'intérieur et, particulièrement, une fronde de fougère Pteridium esculentum associée à une feuille séchée. Chacun de ces éléments avait un sens bien particulier qui pouvait être décrypté comme un message codé et que tout homme initié devait savoir comprendre en fonction des relations totémiques des uns et des autres (Leenhardt, 1930 : 49-51).

Dans les procédés de communication non verbale, il nous faut bien distinguer les éléments cachés de ceux exposés à la vue de tout un chacun. Les fougères ligaturées sur les casse-têtes faisaient donc partie des éléments cachés soigneusement enveloppés, dans certains cas, ou exposés à la vue de tous dans d'autres cas. Mais il est très probable qu'elles ne représentaient qu'un terme du message, le type de matériau utilisé pour les fixer, sa texture, sa couleur et la technique des nouds effectués devaient être d'autres éléments pertinents pour une bonne compréhension. Le père Lambert avait bien saisi la complexité de ces compositions protectrices et porteuse de message (Lambert, $1876: 232$ ).

Après les débuts de la colonisation, les étoffes végétales enveloppant les armes ont été progressivement remplacés par des tissus d'origine européenne $e^{12}$ et on serait tenté de penser que les matériaux constituant ces étoffes avaient moins d'importance que le type de ligature que celles-ci permettaient de réaliser. Ces tissus étaient noués ou enroulés autour du manche et des éléments végétaux, de manière à transmettre un message particulier comme Maurice Leenhardt (1930: 44-45 et pl. XXIII) l'a si bien montré dans les exemples qu'il a eu l'opportunité d'étudier chez les Houaillou.

Emmanuel Kasarhérou (com. pers.), qui a eu l'occasion d'observer la majorité des casse-têtes associés à des fougères et conservés dans les musées, estime qu'il s'agit de la même espèce Pteridium esculentum dont les pinnules sont vraiment très particulières (voir photo 6 ).

Pourtant, comme nous l'avons signalé précédemment Sarasin évoque la présence de Gleichenia sp. à plusieurs reprises sur des casse-têtes du musée de Bâle. Dans la mesure où l'identification de cette fougère est exacte nous pouvons en tirer la conclusion qu'il existait au moins deux types de fougères fixées sur les casse-têtes outre les lycopodes.

Avant de proposer une interprétation de la fonction des ces fougères sur les casse-têtes, interrogeons-nous sur d'autres usages de ces fougères. Comme nous l'avons déjà vu, les fougères n'étaient pas uniquement associées à des objets, elles pouvaient également faire partie des parures corporelles masculines. Moncelon et Sarasin notent ainsi leur présence sur l'enveloppe pénienne des Kanak sans donner de précisions sur le type de fougères :

«Un autre foulard lié le premier à la rosette, à la naissance du pénis, et le tout est souvent entouré de touffes de fougères et d'herbes diverses. " (Moncelon, $1886: 351)$

"L'abondant revêtement du pénis formait un assemblage long et épais et pour rendre l'organe encore plus impressionnant, on entourait l'ensemble de grandes touffes d'herbe et de feuilles de fougères. » (Sarasin, 2009: 151)

Des hommes porteurs de tels feuillages suspendus devant leur étui pénien sont effectivement visibles sur diverses photos d'Allan Hughan (Leenhardt, 1930 : pl. XI ; Kakou, 1998: 89 ; Brianchon, 2007 : 102). Dans la langue paicî, le mot gö signifie à la fois le phallus et le casse-tête (Salomon, 2000 : 67). Doit-on alors faire une relation analogique entre cette pratique et celle consistant à envelopper le manche des casse-têtes de forme phallique avec des végétaux comme marque de virilité et de force ? Cela est fort probable, mais il est difficile de savoir quelles étaient les significations et les circonstances exactes du port de cette parure.

Certains types de fougères auraient donc été cognitivement associés à la force virile et à l'exaltation du combat guerrier et ces végétaux ligaturés sur les casse-têtes auraient vraisemblablement eut une fonction magique, comme l'écrit Françoise Girard :

"Leur efficacité est souvent renforcée par des herbes magiques. » (Girard, $1953: 303$ )

Ce qui correspond à l'indication inscrite sur la fiche d'un casse-tête du musée de Quimper (cf. supra). Le discours de guerre prononcé par le grand chef Mindia (cf. supra et photo 7) lors d'une insurrection en 1878 est très significatif en ce sens.

" Je parlerai jusqu'au bout, à la bouche de ce peuple qui là-bas danse, remue, pousse des cris, déborde, s'agite, et ressemble à un fourmillement de grillons; sa foule se presse, ses visages s'exaltent, alors qu'ici résonne en bruit de pleurs et de heurts l'entrechoquement des sagaies, casse-têtes et becs de tortue. Vous danserez, portant sur l'épaule toutes les armes gardées chez vous et fraîchement ornées de feuillage sacré." (Leenhardt, $1932: 313-314)$

12. Les premiers tissus européens acquis par les Kanak provenaient d'échanges avec les marins des navires des premières expéditions ayant fait escale sur les côtes de Nouvelle-Calédonie à la fin du XVIII ${ }^{\mathrm{e}}$ siècle et au début du XIX ${ }^{e}$ siècle. À leur suite, les santaliers ont importé de nombreuses cotonnades, si bien qu’après les débuts de la colonisation, dans les années 1870 , les Kanak disposaient déjà d'un grand choix de tissus aux couleurs diverses. 
Leenhardt rapporte également (1930: 156157) que lors de la distribution des vivres aux invités durant un pilou, la tête des ignames de taille moyenne placées en dernier au dessus du tas de produits accumulés était ornée d'un cornet de fibre de cocotiers ou d'un morceau de turban, ou encore d'un bagayou (étui pénien). Il précise, dans une note, que cette extrémité supérieure était donc considérée tantôt comme une tête, tantôt comme un phallus. Lors de la présentation de la récolte d'ignames aux groupes utérins, l'orateur portant un casse-tête sur l'épaule évoquait l'herbe de guerre, celle qui donne la chaleur au guerrier et il les remerciait pour les ignames qu'ils avaient apportées dans leurs ornements de feuilles et de fougères, avec la conque semblable à celle du faîte de la case (ibid., 1930 : 153-154). Selon Isabelle Leblic :

"dans l'aire paicî, la "liane fougère" nyärä-èdërë (non identifiée) est toujours associée à la cuisson de la première igname; [...] c'est aussi un médicament ${ }^{13}$ pour certains lignages, comme les Näaucùùwèè Görötädo. [...] Cette fougère est également utilisée pour entourer les aliments remis de manière coutumière aux neveux, aux petits frères, aux tantes et aux sœurs » (Leblic, 2010 et com. pers.)

Il est permis de penser qu'il existait une relation entre phallus, casse-tête et ignames dans le monde kanak ancien comme cela existait chez les Iatmul et les Abelam de Nouvelle-Guinée, entre phallus, flûte et ignames (Bateson, 1971; Coiffier, 1994 : 873-874). Cette relation se situait vraisemblablement dans le domaine de la fertilité et la fécondité. Il existe des informations assez précises concernant les usages d'une des deux fougères évoquées ci-dessus.

"Cette fougère est commune à Art parce que les terrains qu'elle occupe sont généralement stériles [...] dans les plateaux des montagnes à terre rouge, j'ai souvent observé qu'elle se couvre de manne $e^{14}$. Les naturels en mangent la racine après l'avoir fait légèrement torréfier [sic]. " (Montrouzier, 1860, cité par Suprin, $2011: 75)$

Dans la région de Houaillou, les fougères fixées sur les manches des casse-têtes étaient appelées bwâwè, bawe ou boawe en langue ajïe, boè en paicî et pet ou pat en jawé (Suprin, 2011 : 75). Ces noms désignent une espèce de "fougère aigle" commune sur les terrains serpentineux les plus pauvres; Pteridium esculentum G. Forst. Cockayne (Cronin, 1989: 164-165) dont les frondes de couleur vert foncé sont rigides et de formes deltoïdes. Selon le dictionnaire de la langue ajië, cette fougère était utilisée comme symbole des circonstances solennelles (deuil, guerre, ...) et le long des chemins les indigènes repliaient la feuille sur la tige pour indiquer une nouvelle grave. Elle était liée au karoti [karöti] et au wesara [wêyârâ], perches qui ferment les sentiers menant vers le mort, pour en défendre l'entrée à partir du quatrième et cinquième jour de son exposition; elle était liée au manche des armes et indiquait la guerre; posée sur une branche en travers d'un sentier, elle fermait la voie (Lercari, 2002). Leenhardt rapporte le fait de la construction par un Kanak christianisé d'un wesara avec des fougères accrochées à la barre transversale placée entre deux poteaux, ce qu'il perçoit comme une première manifestation d'art et de symbolisme pagano-chrétien (1935: 354-355). Cette fougère était également utilisée pour soulever le rebord des toitures. Son rhizome rampant est recouvert de poils roux comme ceux des roussettes. Les frondes de cette fougère prennent en séchant, sur certains terrains, une légère couleur argentée du plus bel effet et elles deviennent alors extrêmement inflammables. La présence de composés à base de silice dans les tissus des frondes sèches de la fougère-aigle Pteridium esculentum les faisait utiliser, jadis, comme abrasif pour poncer les manches des casse-têtes (Sarasin, 2009: 172).

Il semblerait donc que diverses fougères, outre leurs usages alimentaires et médicaux, furent associées à la guerre dans diverses régions de Nouvelle-Calédonie. Ces fougères auraient condensé les énergies vitales dans un contexte local où les feux de brousse sont particulièrement fréquents. Les Kanak auraient bien perçu dans ces végétaux tout le potentiel dévastateur, stimulé par le feu et en auraient tiré un concept similaire à celui de l'oiseau phénix si important dans d'autres sociétés ?

Cependant, les écrits du docteur Jean-Baptiste Vincent sont les seuls à présenter une version différente de l'utilisation de ces fougères :

«J'allais de Ponérihouen à Houaïlou, en suivant les sentiers muletiers, lorsque j'aperçus une longue théorie de Canaques. Ils venaient d'un pilou et portaient des armes. Je m'informai auprès du chef si je pouvais acheter des casse-têtes; je m'en procurai un d'immolateur; je payai et je serrai la main du chef. Nous nous quittions tous satisfaits. Au moment de partir, j'ôtai les fougères qui entouraient mon cassetête et les jetai, parce qu'elles m'empêchaient de bien tenir mes guides. Aussitôt un $k a^{16}$ ! formidable

13. Les médicaments wâi sont en effet liés à un clan précis et à un totem particulier.

14. La manne est le nom donné à toutes sortes d'exsudats qui suintent de diverses parties des plantes.

15. NDLR. - Selon Jean-Claude Rivierre (1983 : 48), boè est « 1 . fougère : Pteridium aquilinum (L.) Kuhn, Dennstaedtiacé ; 2 . arbre de forêt ; 3 . figure de jeu de ficelle ; 4. palissade, barrière autour des présents".

16. Ka exprime la désapprobation violente, la colère. Le $k a$ ajië de Néavin devient $t c h a, k a r . .$. dans d'autres tribus et d'autres langues. Le contraire de ka est naheu en ajië (Vincent, 1895 : 85). 
s'éleva. Je me détournai pour constater ce qu'avaient les indigènes : je les vis me regarder méchamment et protester clairement contre ce que je venais de faire. J'appris, le soir, qu'en parcourant toute tribu qui n'est pas la leur, les Canaques entourent leurs casse-têtes de feuilles (généralement des feuilles de fougères); ôter ces feuilles, c'est commettre un acte d'hostilité. " (Vincent, 1895 : 84-85)

Cette information est reprise par Sarasin (2009 : 174), qui écrit qu'on ne revêt ces cassetêtes de feuilles que lorsqu'on traverse paisiblement le territoire d'une tribu étrangère et il en déduit que cette coutume aurait été un signe de paix. Il est possible que J.-B. Vincent ait été abusé par ces informateurs ou qu'il ait interprété leurs explications. Mais, ces propos permettent de mesurer l'importance accordée à ces fougères par les Kanak.

\section{Conclusion}

Nous constatons que la majorité des casse-têtes associés à des bouquets de végétaux sont rentrés dans les collections des musées après l'insurrection kanak de 1878, soit dans les années 1880 1890. Il est difficile d'établir, un siècle et demi après l'achat ou la confiscation de ces armes à leurs propriétaires, quelle était la fonction réelle de ces bouquets végétaux parfois ligaturés sur leur manche. Les recherches menées dans les collections permettent cependant de mettre en évidence l'utilisation d'un nombre très restreint d'espèces de fougère, vraisemblablement deux, ligaturées sur les casse-têtes étudiés. Il serait nécessaire néanmoins que tous les spécimens de fougère associés à des objets Kanak dans les musées cités ( $c f$. supra) puissent être authentifiés par des spécialistes des Ptéridophytes. Quoi qu’il en soit, nous ne saurons sans doute jamais, si la présence de ces feuillages sur les casse-têtes des hommes paradant fièrement devant le photographe Allan Hughan, indiquait une intention belliqueuse ou pacifique. Mais, il y a très peu de chance que ces différents signes aient été compris par les colons et les soldats de l'époque. Les armes furent confisquées et les contrevenants souvent tués sans égard par les nouveaux envahisseurs. Deux sociétés commencèrent ainsi à vivre côte à côte sans se comprendre, ni par le langage, ni par leurs codes.

\section{Remerciements}

Emmanuel Kasarhérou (chargé de mission pour l'Outre-Mer auprès du Directeur du département du patrimoine et des collections du musée du quai Branly) a recueilli depuis plusieurs années dans les musées de France et européens une énorme documentation photographique relative aux objets kanak. Dans le cadre du projet L'inventaire du patrimoine kanak dispersé, il a eu l'occasion de faire ces visites pour préparer l'exposition sur l'art kanak qui doit ouvrir, à la fin 2013, au musée du quai Branly. Nous tenons à le remercier pour nous avoir aimablement transmis de nombreuses informations personnelles et une des photographies qui illustre cet article. D'autre part, nos remerciements vont également à Claude-Alain Künzi (conservateur du musée de Saint-Imier), Claire Brizon et Boël Sartor (musée des Confluences de Lyon), Sylviane Bonvin Pochstein et Frédérique Gaillard (muséum d'histoire naturelle de Toulouse) qui nous ont gracieusement fourni diverses illustrations, à Roland Kaehr (conservateur honoraire du musée de Neuchâtel), Jacqueline de la Fontinelle et Isabelle Leblic pour les informations communiquées ; enfin à Pierre Cabalion (botaniste à l'IRD de Nouméa, membre de la Société française d'ethnopharmacologie [SFE] et président de l'Association pour la promotion des plantes aromatiques et médicinales en Nouvelle-Calédonie [APPAM-NC]) qui a pris la peine de relire cet article et d'en corriger certaines erreurs botaniques. C'est également lui qui a vérifié et complété l'index.

\section{BIBLIOGRAPHIE}

BATEson Gregory, 1971 (1936). La cérémonie du naven, les problèmes posés par la description sous trois rapports d'une tribu de Nouvelle-Guinée, Paris, Editions de Minuit.

Boulay Roger, 1993. Le bambou gravé kanak, Marseille/Nouméa, Éd. Parenthèses/ADCK, coll. Arts témoins.

Brianchon Alain, 2007. Art d'Océanie. Art of Oceania, Nouméa, Éditions Footprint Pacifique.

CoIffier Christian, 1994. L'écorce et la moelle du rotin, tshimbe kuvu, kwiya kuvu, conception iatmul de l'univers, thèse de doctorat, Paris, EHEss.

Cronin Leonard, 1989. Key Guide to Australian Palms Ferns and Allies, Frenchs Forest, NSW, Reed Books

Duuren David van et Tristan Mostert, 2007. Curiosities from the Pacific Ocean, (van) Leiden, Amsterdam and C. Zwartenkot Art Books, Tropenmuseum.

Girard Françoise, 1953. Les riches collections néo-calédoniennes du musée de l'Homme, Journal de la Société des Océanistes IX, 9, pp. 302-306. 
Guiart Jean, 1987. Mythologie du masque en Nouvelle-Calédonie, Paris, Société des Océanistes, Publication de la Société des Océanistes 18.

Kakou Serge, 1998. Découverte photographique de la Nouvelle-Calédonie (1848-1900), Paris, Actes-Sud.

KasArhÉrou Emmanuel, 1990. Le masque, in Roger Boulay, De jade et de nacre. Patrimoine artistique kanak, Paris, RMN, pp. 143-151.

—, 1993. LE Masque kanak, Marseille-Nouméa, Éd. Parenthèses-ADCK, Coll. Arts témoins.

Kasarhérou Emmanuel, Béalo Wedoye, Roger Boulay et Claire Merleau-Ponty, 1998. Guide des plantes du chemin kanak, Nouméa, Agence de développement de la culture kanak.

La Billardiere Jacques Julien Houtou (de), 1799. Atlas pour servir à la relation du voyage à la recherche de La Pérouse, Paris, H. J. Hansen.

Lambert Pierre, 1976 (1900). Mours et superstitions des Néo-Calédoniens, Nouméa, Société d'études historiques de la Nouvelle-Calédonie, Publications de la SEHNC 14.

Leburc Isabelle, 2010. «Échanger dans la route ». Parenté, alliance et territoire à Ponérihouen (Nouvelle-Calédonie), manuscrit inédit présenté dans le cadre de l'HDR, EPHE, Paris (à paraitre).

Leenhardt Maurice, 1930. Notes d'ethnologie néo-calédonienne, Paris, Institut d'ethnologie, Travaux et mémoires viII.

—, 1932. Documents néo-calédoniens, Paris, Institut d'ethnologie, Travaux et mémoires Ix.

-, 1935. Vocabulaire et grammaire de la langue houaillou, Paris, Institut d'ethnologie, Travaux et mémoires $\mathrm{X}$.

Lercari Claude, 2002. Dictionnaire ajië-français à l'usage des étudiants, Nouméa, Centre de documentation pédagogique de NouvelleCalédonie.

Lobsiger-Dellenbach Marguerite, 1967. Dix bambous gravés néo-calédoniens du musée des Arts africains et océaniens (Paris), Journal de la Société des Océanistes xxiII, 23, pp. 59-99.
LuQueT Georges-Henti, 1926. L'art néo-calédonien, Documents recueillis par Marius Archambault, Paris, Institut d'Ethnologie, Travaux et Mémoires II.

MonCelon Léon, 1886. Réponse, alinéa par alinéa, pour les Néo-Calédoniens, au questionnaire de sociologie et d'ethnographie de la société, Bulletin de la Société d'Anthropologie de Paris 9, pp. 345-380.

Montrouzier Xavier, 1860. Notice historique, ethnographique et physique sur la Nouvelle-Calédonie, Paris, impr. Ch. Lahure.

Musée des Beaux-Arts de Chartres, 2009. L'art ancestral des Kanak, Chartres, Musée des Beaux-Arts.

Riviere Jean-Claude, 1983. Dictionnaire paicîfrançais, suivi d'un lexique français-paicî, $\mathrm{Pa}-$ ris, SÉLAF, Langues et cultures du Pacifique 4.

Salomon Christine, 2000. Savoirs et pouvoirs thérapeutiques kanaks, Paris, Inserm/Presses universitaires de France

SARASIN Fritz, 2009 (1929). Ethnographie des Kanak de Nouvelle-Calédonie et des îles Loyauté (1911-1912), Paris, Ibis Press.

Smith B.L., P.P. Embling, M.P. Agnew, D.R. Lauren et P.T. Holland, 1988. Carcinogenicity of bracken fern (Pteridium esculentum) in New Zealand, New Zealand Veterinary Journal 36, 2, pp. 56-58.

Suprin Bernard, 2011. Florilèges des plantes en Nouvelle Calédonie, tome 1, Nouméa, Éditions Photosynthèse.

Tjibaou Jean-Marie, 1976. Recherche d'identité mélanésienne et société traditionnelle, Journal de la Société des Océanistes XxxiI, 53, pp. 281-292.

Vieillard Eugène et Émile Deplanche, 1862 et 1863. Essais sur la Nouvelle-Calédonie, Paris, Revue maritime et coloniale 6 et 7, republié en fac-similé en 2005, Paris, L'Harmattan.

VInCENT Eugène J.B.M., 1895. Les Canaques de la Nouvelle-Calédonie. Esquisse ethnographique, Paris, Angustin Challamel. 


\section{Annexe I : Index botanique des Ptéridophytes de Nouvelle-Calédonie}

Sont présentés ici ceux cités dans le texte avec les noms de synonymes et les noms de famille. Depuis plus d'un siècle, les noms scientifiques de nombreuses espèces de fougère ont été révisés, ce qui implique parfois des appellations nouvelles.

\begin{tabular}{|c|c|c|}
\hline Nom d'espèce & Synonyme & Famille \\
\hline \multicolumn{3}{|c|}{ Fougères } \\
\hline Alsophila vieillardii (Mett.) R. M. Tryon & Cyathea vieillardi Mett. ${ }^{17}$ & \multirow[b]{2}{*}{ Cyatheaceae } \\
\hline Alsophila sp. & cf. Cyathea sp. & \\
\hline Angiospteris evecta (J.G. Forst. Hoffm. & & MARATTIACEAE \\
\hline Asplenium nidus $\mathrm{L}$. & & ASPLENIACEAE \\
\hline Blechnum gibbum (Labill.) Mett & & \multirow{2}{*}{ BLECHNACEAE } \\
\hline Blechnum orientale L. & & \\
\hline Dicksonia thyrsopteroides Mett. & & DICKSONIACEAE \\
\hline \multirow{3}{*}{ Dicranopteris linearis (Burm. F.) Underw. } & $\begin{array}{l}\text { Gleichenia linearis (Burm. F.) } \\
\text { C.B.Clarke }\end{array}$ & \multirow{4}{*}{ Gleicheniaceae } \\
\hline & Mertensia dichotoma (Thunb.) Willd. & \\
\hline & Gleichenia dichotoma (Thunb.) Hook. & \\
\hline Gleichenia dicarpa R. Br. & Platyzoma dicarpium (R. Br. Desv.) & \\
\hline Helminthostachys zeylanica (L.) Hook. ${ }^{18}$ & & OpHIOGLOSSACEAE \\
\hline Lomaria sp. & cf. Blechnum sp. & BLECHNACEAE \\
\hline Lygodium reticularum Schkuhk. & & $\begin{array}{l}\text { LYGODIACEAE } \\
\text { ou SCHIZAEACEAE }\end{array}$ \\
\hline Marattia sp. & & Marattiaceae \\
\hline Polypodium phymatodes Linn. & & Polypodiaceae \\
\hline $\begin{array}{l}\text { Pteridium esculentum subsp. esculentum G. } \\
\text { Forst. Cockayne }\end{array}$ & & Dennstaedtiaceae \\
\hline \multicolumn{2}{|l|}{$\begin{array}{l}\text { Sticherus brackenridgei (E. Fourn.) H. St } \\
\text { John }\end{array}$} & \multirow{5}{*}{ GLeicheniaceae } \\
\hline \multirow{2}{*}{ Sticherus flabellatus (R. Br.) H. St. John } & Gleichenia flabellata R. Br. & \\
\hline & Gleichenia flabellaris & \\
\hline \multicolumn{2}{|l|}{ Sticherus montaguei (Compton) Natai } & \\
\hline \multicolumn{2}{|l|}{ Stromatopteris moniliformis Mett. } & \\
\hline \multicolumn{3}{|c|}{ Lycopodes } \\
\hline Lycopodiella cernua (L.) Pic. Serm. & Lycopodium cernuиm L. & \multirow{4}{*}{ LYCOPODIACEAE } \\
\hline \multirow{3}{*}{ Huperzia phlegmaria (L.) Rothm. } & Huperzia mirabilis (Will.) Holub & \\
\hline & Lycopodium phlegmaria L. & \\
\hline & Lycopodium mirabile Willd. & \\
\hline
\end{tabular}

17. N.B. Espèce autrefois citée "vieillardi ", aujourd'hui standardisée en "vieillardii " comme la plupart des noms d'espèce forgés sur un nom masculin, comme Vieillard.

18. Le genre est monotypique et disparaît dès lors que l'espèce devient syn. de Tectaria zeylanica (Houtt.) Sledge. 


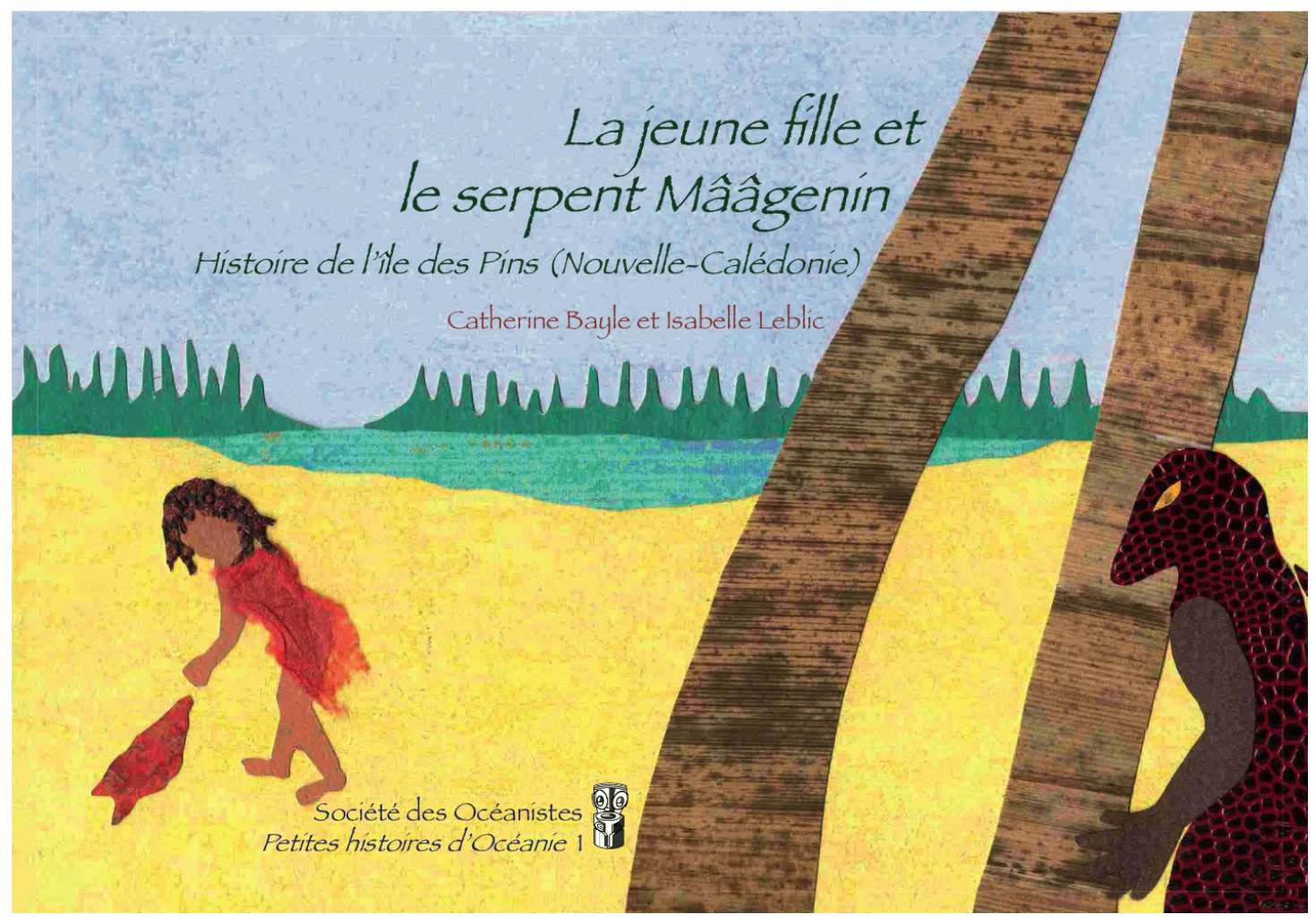

$2011-9,50 €$.

En vente sur http://oceanistes.org/oceanie/spip.php?rubrique29 avec paiement en ligne 